\title{
Studying of Lactulose Hygroscopicity and Microstructure after Spray Dehydration
}

\author{
ANDREI LISITSYN ${ }^{1}$, OKSANA KUZNETSOVA ${ }^{2}$, \\ MIKHAIL MINAEV ${ }^{2}$ and ALEXANDER PROSEKOV ${ }^{2}$
}

\begin{abstract}
${ }^{1}$ Federal State Scientific Institution "All-Russian Research Institute of Meat Industry.
VM Gorbatov"Talalikhina 26, Moscow, 109316, Russia.

${ }^{2}$ Federal State-owned Budgetary Educational Institution of Higher Vocational Education

"Kemerovo Institute of Food Science and Technology" Stroiteley Boulevard, 47, 650056,

Kemerovo, Russia.

${ }^{*}$ Corresponding author E-mail: mtrushin@mail.ru
\end{abstract}

http://dx.doi.org/10.13005/ojc/320212

(Received: February 18, 2016; Accepted: March 23, 2016)

\section{ABSTRACT}

Investigations in the present work were directed towards the study of the dehydration temperature influence on the mass fraction of moisture in lactulose. The moisture mass fraction index is the most important to estimate the quality of primary, semi-finished and finished products. Resulting from this, investigations have been directed onto the study of the dehydration temperature influence on the mass fraction of moisture in the solution. A technology of getting dry lactulose by way of spray dehydration has been developed. It has been proved that while the temperature of dehydrating a lactulose solution with the mass fraction of $50 \%$ rises, the moisture content in the product decreases. The lactulose powder quality was estimated by such factors as mass fraction of moisture, particle size, solubility index and hydroscopicity, as these factors produce the most influence upon consumer properties of the products. The data received show growth of the lactulose solubility index with the particle size increase. This is evidently connected with the fact that a big size of the particles makes interaction between molecules of water and lactulose more difficult as well as forming donor and acceptor (hydrogen) bonds, and consequently the solution process.

Keywords: lactulose, solubility, powder quality.

\section{INTRODUCTION}

Lactulose is a disaccharide possessing useful physiological properties and used actively in different industries. It is worth noting that a whole number of investigations carried out for the last few years have proved a possibility of successful lactulose usage within feed additives used in different fields'.

The most widely spread method used to get finely-divided food and pharmaceutical products 
is spray dehydration. It may be carried out within a large range of working temperatures, the process is characterized by a short time of the material contact with the hot heat conductor and high productivity by the evaporated moisture ${ }^{2}$.

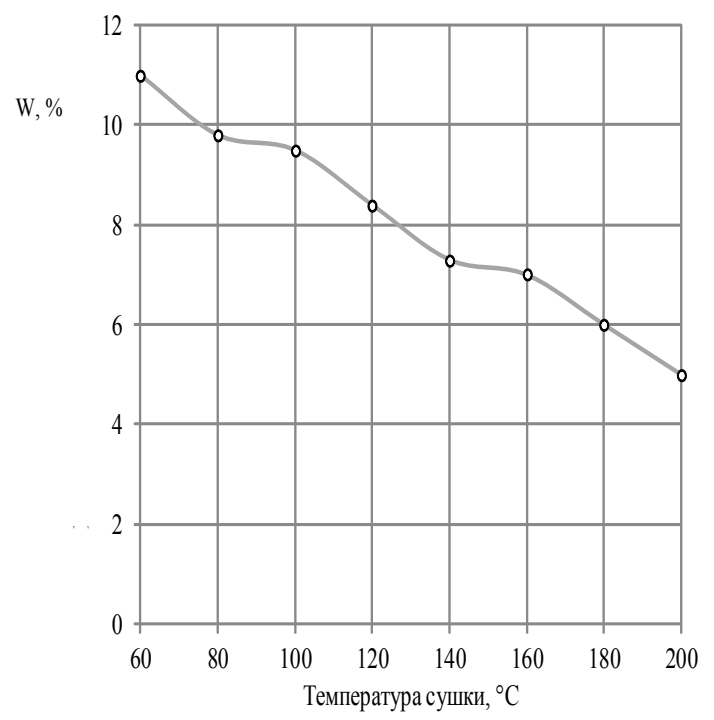

Vertical: Mass fracture of moisture in the product, \% Horizontal: Dehydration temperature, ${ }^{\circ} \mathrm{C}$

Fig. 1: Change of moisture mass fraction in the product depending on the dehydration temperature at lactulose solution mass fraction of $50 \%$

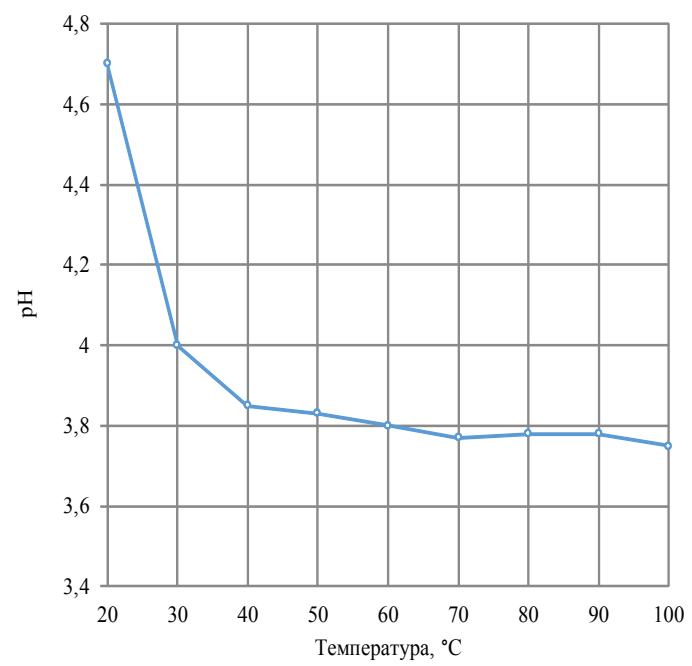

Fig. 2: Dependence of lactulose solution $\mathrm{pH}$ value on the temperature of a sample heating
The quality of the dehydrated products, especially thermolabile ones, often depends very much not on the kinetics of the moisture removing, but on the kinetics of their heating during the process of dehydration. It is caused by a strong, usually exponential dependence of the chemical, physicochemical and structure rheological transformations taking place during the dehydration on the temperature of the material being dehydrated ${ }^{3}$.

The product received by spray dehydration has a number of new properties (dispersity, chemical activity, adsorbing capacity, whether the particles charged or not, etc.) characteristic of solid dispersed materials. Sensitivity of products to the temperature influence and its duration may lead to undesirable changes that will effect the end-product quality. Durable thermal exposure causes not only negative changes in the product, but may also result in its selfcombustion and increasing the end-product loss ${ }^{4}$.

Lactulose dry powders are supposed to be more effective in usage. By the present time, a number of techniques of getting lactulose by different dehydrating methods have been patented. The main problem of the existing techniques of lactulose solutions spray dehydration is the presence of expensive binding components - catalysts and stabilizers - used to decrease hygroscopicity and humidity of the end-product as well as to accelerate the dehydrating process. An important stage, increasing significantly the cost-price of the endproduct, is the clearance from these components which reduces the lactulose mass fraction and the powder received exhibits high hygroscopicity ${ }^{5}$.

Taking this into account, development of the technique of receiving dry lactulose by the method of spray dehydration is timely and prospective. The purpose of the present work is investigation and development of the technique of receiving dry lactulose by the method of spray dehydration.

\section{METHODS}

The following items were investigation objects at different stages of the work: 
Solutions with the lactulose mass fraction of $20-60 \%$ (OOO "Shekhon-Lactulose", Russia);

distilled water corresponding to GOST (= State Standard) 6709-72 "Distilled Water. Technical Specifications";

To get lactulose solutions with different concentration, a solution with the lactulose mass fraction $50 \%$ corresponding to TU (= Technical Specification) 9229-003-39185375-2003 was taken and diluted to get solutions with the lactulose mass fraction of $20-40 \%$ and condensed to get a solution with the lactulose mass fraction of $60 \%$.

- $\quad \mathrm{N}$-trimethylsilylmidazol (Panreac, Spain);

- hexane (NPK "KRIOCHROME", Russia);

- potato agar (FSZ (= Federal Service of Health Care) 2009/03706);

- $\quad$ beef-extract agar (GOST 20730-75);

- malt agar (FSZ 2009/03709);

- Endo medium (TU 9229-072-00419785-97).

Other used domestic and imported reagents had a grade not lower than CP. When executing the works, generally accepted, standard and original investigation methods were used.

Selection of test samples and their preparation for the analysis were performed in accordance with GOST 9225, GOST 26809, GOST 26929.The dry substance mass fracture in the lactulose solutions was determined by the refractometric method in accordance with GOST 24908-84.

The mass fraction of lactulose and other carbohydrates in the solutions and dry lactulose was determined by the method of gas-liquid chromatography (GLC) with GCMS-QP2010 Ultra (Shimadzu, Japan) chromatograph.

The method of gas-liquid chromatography is based upon transformation of saccharides into volatile trimethylsilyl derivatives with their subsequent separation at the chromatograph column and determination with a flame ionization detector.

The summary of the method is as follows: the sample under analysis, preliminarly dried and defatted, was treated by $\mathrm{N}$-trimethylsilylmidazol at 60-70 ${ }^{\circ} \mathrm{C}$ during 1-2 hours. Then an exact amount of hexane was added to the mixture, the hexane surplus hydrolysed with water, after which an aliquot of the hexane phase was injected into the chromatograph. The carbohydrates separation was made on the packed column with the polar phase in an isothermal mode. Monosaccharides came out with the solvent front, lactose came out as two peaks corresponding to alpha- and beta-anomers, and lactulose came out as one peak.

During the experiment, the carrier gas from the cylinder in case of increasing pressure comes continually into the preparation unit, where it gets additional cleaning. The sample input device is a flow-through thermostated independently cylinder chamber. The test sample under analysis (1-10 microlitres) was put into the gas flow at an elevated temperature with an automatic dosing unit through a rubber heat-resistant membrane; the liquid test sample quickly evaporates and is transferred with the gas flow into the chromatograph column placed in the thermostat. The separation was performed at $20-400{ }^{\circ} \mathrm{C}$. Sometimes packed columns $0.5-5$ $\mathrm{m}$ long and $0.2-0.6 \mathrm{~cm}$ in diameter are used for analytical separation. The media is a hard sorbent with a developed surface $\left(50-500 \mathrm{M}^{2} / \mathrm{r}\right)$. The recorder registers the signal change with time.

The quantitative content of lactulose, lactose and other carbohydrates present in the sample was calculated by the method of internal standard, using the preliminarily performed calibration.

Change of a lactulose solution actual acidity under the impact of a heat treatment was determined in the present work. The end-product quality was estimated by such factors as the particle size, humidity, solubility index and hygroscopicity.

\section{RESULTS}

Estimation of the end-product quality indexes is an important stage of investigation that determines efficiency of parameters and methods used to get the end-product. Every product has its own set of indexes that depends on the product purpose, conditions of its production and exploitation and many other factors ${ }^{6}$. 
The set of indexes is finally formed at the product designing stage, as they are built into the construction here. Later on, at the production stage, these indexes get their implementation. At the exploitation (consumption) stage the indexes become the product individual characteristics, distinguish it from other types of products (goods), make its consumer properties and, consequently, make it attractive and competitive ${ }^{7}$.

A desire to consider as many indexes as possible in an attempt of characterizing the product most fully makes the design problem practically unsolvable. It is important to distinguish main

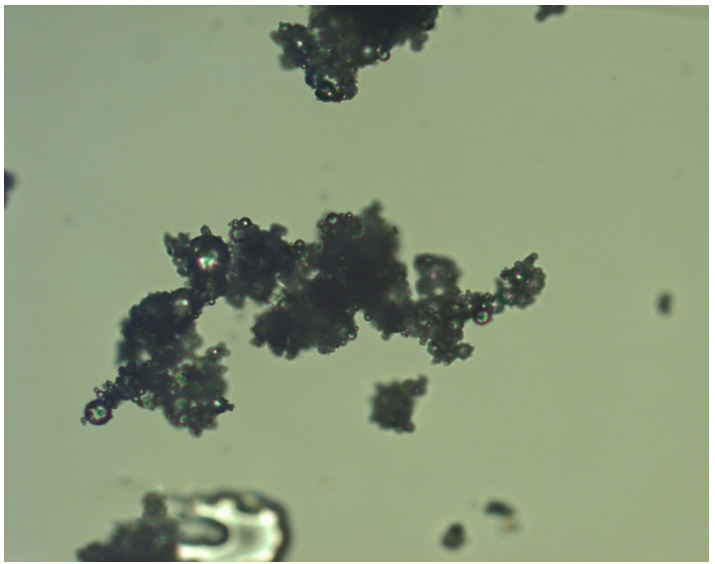

Fig. 3: Microstructure of lactulose powder received at the dehydration temperature of $100 \pm 1^{\circ} \mathrm{C}$ (magnification ratio 200 times)

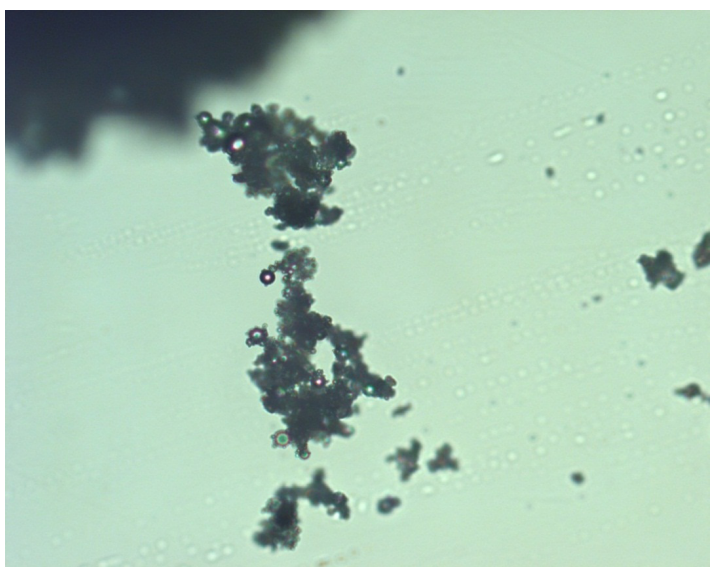

Fig. 4: Microstructure of lactulose powder received at the dehydration temperature of $120 \pm 1^{\circ} \mathrm{C}$ (magnification ratio 200 times) indexes, reflecting the most significant consumer properties of an object. One should also bear in mind that there are obligatory indexes to be considered under certain production and exploitation conditions. It refers mainly to safety, when minimum acceptable level of requirements is established by statutory documents the federal authorities executing control of products quality and safety, such as Russian Technical Supervisory Authority, Federal Service on Customers' Rights Protection, etc. Also in case the product is destined for certain people or can be sold to them somehow, it must meet additional requirements, established by the Russian Federation Law "About Consumers' Rights Protection"8.

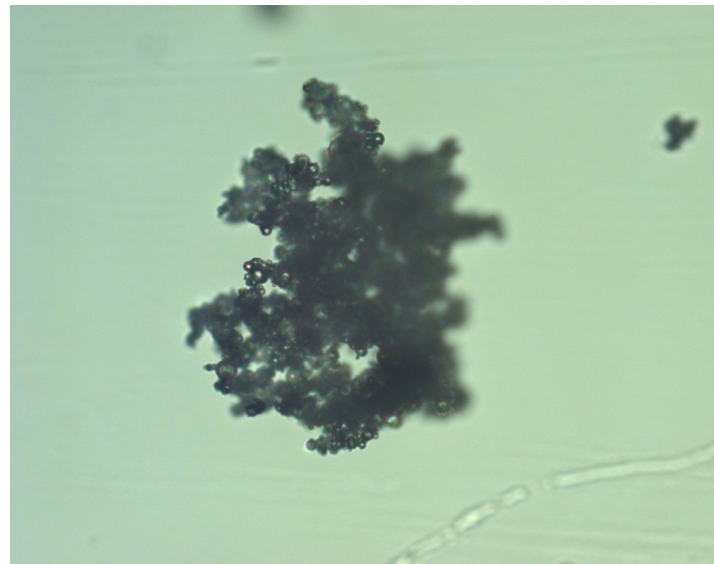

Fig. 6: Microstructure of lactulose powder received at the dehydration temperature of $150 \pm 1{ }^{\circ} \mathrm{C}$ (magnification ratio 200 times)

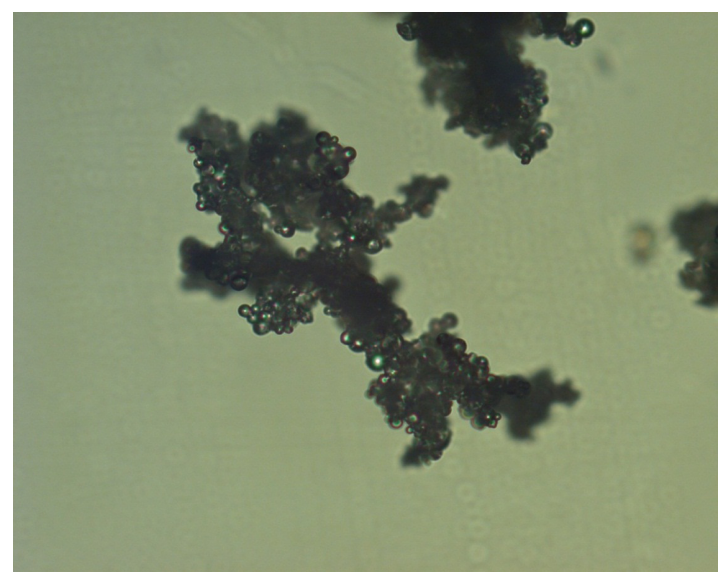

Fig. 5: Microstructure of lactulose powder received at the dehydration temperature of $140 \pm 1^{\circ} \mathrm{C}$ (magnification ratio 200 times) 
The lactulose powder quality was estimated by such factors as mass fraction of moisture, particle size, solubility index and hydroscopicity, as these factors produce the most influence upon consumer properties of the products.

The moisture mass fraction index is the most important to estimate the quality of primary, semi-finished and finished products. Resulting from this, investigations have been directed onto the study of the dehydration temperature influence on the mass fraction of moisture in the solution (Figure 1).

Dependence of actual acidity change on the temperature of a dry lactulose sample heating is shown in Figure 2. Powder-like food stuffs are two-phase systems where hard particles of the dispersed phase are distributed in the gas (air) dispersion medium and are characterized by a large interfacial area. Existence of this area determines the most important technological properties of powders, including the following ones: flowability determined by the value reciprocal to viscosity; compactability characterized by the powder volume change under dynamic load (clamminess); caking while being stored caused by forming structures whose strength exceeds the primary one.

Caking and lumping are based upon processes of cross-linking caused by spontaneous connection of the dispersed phase particles into three-dimensional structures. Two main causes may be distinguished as a base for such spontaneous connection of particles in powder-like products ${ }^{9}$.
The first cause of caking and lumping of lactulose water-soluble powders is appearing of accreting bridges between the powder particles due to its humifying during a long storage in the open air, the other one is increasing the area of contact between the particles due to the plastic yield under the influence of overlying layers weight ${ }^{10,11}$.

Caking and lumping of powder-like food stuffs result in reduction of flowability and deterioration of their consumer properties, and in the extreme case in the total loss of the powder quality $^{12,13}$.

To ensure the necessary flowability of food powders during the whole specified shelf life, hard highly dispersed additives are put into them that absorb moisture or prevent from increasing the area of contact between the particles. To avert caking of hygroscopic powders, hydrophobization of the particles surface with surface-active agents is also used. Molecules of the surface-active agent, being adsorbed onto the surface of hard particles, cover them with a thin membrane that forms a barrier for the moisture evoking caking and lumping ${ }^{14,15}$.

Such additives often increase significantly the cost-price of the end-product. In case of prebiotic products, lactulose is referred to such products, application of such substances is unacceptable as they reduce used properties of the product ${ }^{16}$.

This was the purpose of studying the dry material microstructure during the investigation. Microstructure of lactulose powders received by the

Table 1: Solubility index, particle size, hygroscopicity of the dry lactulose received from solutions with different concentration

\begin{tabular}{lccc}
\hline $\begin{array}{l}\text { Lactulose } \\
\text { mass fraction } \\
\text { in the solution, } \\
\%\end{array}$ & $\begin{array}{c}\text { Particle } \\
\text { size, } \boldsymbol{\mu m}\end{array}$ & $\begin{array}{c}\text { Solubility } \\
\text { index, cm3 of } \\
\text { wet residue }\end{array}$ & $\begin{array}{c}\text { Hygroscopicity, } \\
\%\end{array}$ \\
\hline & & \\
40,0 & $8,0 \pm 2,0$ & $0,20 \pm 0,01$ & $10,0 \pm 0,4$ \\
50,0 & $12,0 \pm 3,0$ & $0,30 \pm 0,03$ & $5,0 \pm 0,2$ \\
60,0 & $20,0 \pm 5,0$ & $0,30 \pm 0,03$ & $1,0 \pm 0,1$ \\
\hline
\end{tabular}


method of spray dehydration at different temperatures is given in figures 3-6.

\section{DISCUSSION}

Water enters inherently into the composition of all sugars, including lactulose. Coming into contact with an energy unsaturated molecule section, water passes into the bound state, which may have a significant impact on the activity of the substance and technological peculiarities of its production and packaging. An active influence of water on properties of the processed raw materials and finished products is evident when the moisture content changes in them. Depending on moisture saturation of the hydrophilic materials, their physical, chemical and technological properties change significantly, that especially shows up when the materials are humified or dehydrated. It is caused by a special character of interaction between water and hydrophilic materials and serves as a basis for developing specific variants of technological operations modes in which change of materials moisture content takes place and, consequently, an active influence of water on the products properties.

The moisture quantity in a product characterizes its energy value, as the more water it contains, the less useful dry substances (protein, fat, carbohydrates, etc.) there are in a mass unit. The product storage stability, its transportability, as well as its suitability for further processing are closely connected with the water content, as moisture excess promotes enzymatic and chemical reactions , activates microorganisms activity, including those causing products spoiling, molding in particular. Due to this fact, moisture content in an object predetermines conditions and period of its storage. Besides, water content in raw materials affects technical and economical indicators of companies' work.

The data given in Table 1 show increase of values of lactulose solubility index as the particle size grows. This is evidently connected with the fact that a big size of the particles makes interaction between molecules of water and lactulose more difficult as well as forming donor and acceptor (hydrogen) bonds, and consequently the solution process. Lactulose received by dehydration of a solution with the mass fraction of $50 \%$ meets the requirements put to food carbohydrates as the solubility index lies within the range from $0,10 \pm 0,02$ to $0,30 \pm 0,02 \mathrm{~cm}^{3}$ of wet residue.

The solubility value for sugars makes up to $0,4-0,5 \mathrm{~cm}^{3}$ of wet residue. Having a low solubility index, a substance will not make a homogeneous mixture when being dissolved. Products with such characteristics are difficult to be used in a usual diet because the powder form, convenient for packing, is not optimal for oral accepting.

Analysis of the curve presented in Picture 1 indicates that as the dehydration temperature of a lactulose solution with the mass fraction of $50 \%$ increases, moisture content in the product reduces. At temperatures from $60{ }^{\circ} \mathrm{C}$ to $100^{\circ} \mathrm{C}$ gradual decrease of moisture mass fraction from $11.0 \%$ to $9.5 \%$ happens.

At temperatures from $100^{\circ} \mathrm{C}$ to $140{ }^{\circ} \mathrm{C}$ sharp decrease of moisture mass fraction from $9.5 \%$ to $7.3 \%$ is observed. Evidently it happens due to reaching the water boiling temperature, which increases abruptly its evaporation speed.

At further temperature increase we come across a range from $140{ }^{\circ} \mathrm{C}$ to $160^{\circ} \mathrm{C}$ where there is practically no change of moisture mass fraction. This effect is connected with the beginning of lactulose caramelization process and moisture binding. Further temperature rise from $160{ }^{\circ} \mathrm{C}$ to $200{ }^{\circ} \mathrm{C}$ causes reduction of moisture mass fraction in the product connected with the caramelization process intensification and lactulose burning.

The investigation results presented in Picture 2 indicate reduction of actual acidity indicator at the processing temperature increase. The initial $\mathrm{pH}$ value of lactulose solution with the mass fracture of $50 \%$ making 4.7 is due to the presence of organic acids, such as lactic acid and in a less degree of acetic and formic acids, in the original solution. The maximum acidity value is observed in a sample not exposed to thermal influence and makes 4.7. The minimum acidity value is observed in a sample processed at $100^{\circ} \mathrm{C}$ and it is 3.75 . The actual acidity 
sharp change from 4.7 to 3.82 starts at the solution processing temperature from $20^{\circ} \mathrm{C}$ to $40{ }^{\circ} \mathrm{C}$. Then a more gradual acidity reduction from 3.82 to 3.78 happens at the temperature from $40^{\circ} \mathrm{C}$ to $70^{\circ} \mathrm{C}$.

This fact is evidently connected with forming lactulose destruction products and moving of the solution $\mathrm{pH}$ towards acid side. The samples presented in Pictures 3 and 4 have crumbly sticky texture. The sample presented in Picture 5 has a crumbly texture, but less sticky than the samples received at the dehydration temperature $100 \pm 1$ ${ }^{\circ} \tilde{N}$ and $120 \pm 1{ }^{\circ} \mathrm{C}$. The sample received at the temperature of $150 \pm 1{ }^{\circ} \mathrm{C}$ (Picture 3 ) a crystal structure due to partial caramelization of the sample. Also the sample has got more free-running texture.

\section{CONCLUSION}

So, the temperature of $140 \pm 1^{\circ} \mathrm{C}$ is optimal for getting lactulose powders under production-line conditions by the spray dehydration method. The sample received under such conditions exhibits the minimal clamminess, which provides for a longer storage period for the end-product without loss of organoleptical properties. By the results of microstructural analysis one can conclude that the lactulose sample received at $150 \pm 1{ }^{\circ} \mathrm{C}$ cannot be considered as a working one, as caramelization of the sample leads to a significant loss of functional and organoleptical properties of the product.

As one can see from Pictures 3-6, dehydrated lactulose samples consist of particles with the size of $7 \pm 0,2 \mathrm{im}$ with smooth surface of round and oval form, which speaks of the homogeneity of the received product.

\section{REFERENCES}

1. Eremina, M.V. Development of concentrated feedstuff technology of functional purpose from dairy protein-and-carbohydrate materials: Candidate of Science: 05.18.04 / Marina Vladimirovna Eremina. - Stavropol. 2008, 157.

2. Zabodalova, L.A. Technicaland Chemical and Microbiological Control at Companies of Dairy Products Industry: Study Guide / L.A. Zabodalova.-St. Petersburg: Troitsky Bridge, 2009, 224.

3. Zakharova, L.M.; Zakharenko, M.A.; Eremina, I.A. Galactooligosaccharides as Factor of Bifidus Bacteria Growth // Dairy Products Industry. 2010, 1, 53-53.

4. Zakharova, L.M.; Lupinskaya, S.M.; Ovchinnikova, T.A. Consumers' Demand for Functional Dairy Products // Dairy Products Industry. 2006, 8, 8-9.

5. Rubin, M.A.; Mikhaylova, A.A.; Sulman E.M. et al. Catalytical Technologies of Lactulose Receiving // Bulletin of Tver State University. Series: Chemistry. 2011, 12, 107-114.

6. Kim, V.V.; Serov, A.V.; Kharitonov, D.V. Physiological Properties of Lactulose // Dairy Products Industry. 2006, 6, 54.
7. Kiselyov, S.V. Lactlose: Second Breath of the Familiar Prebiotic // Milk Processing. 2007, 8, 35-36.

8. Kozlova, N.V. Development of the Technology of Lactulose from High-concentrated Lactose Solutions : Candidate of Science Thesis in Technical Science : 05.18.04 / Natalia Vladimirovna Kozlova.- Stavropol, 2007, 139.

9. Kravets, A.B. Control of Bifidogenic Serum Concentrates Production // Dairy Products Industry. 2008, 11, 46.

10. Kravchenko, E.F. Present Condition and Prospects of Lactoserum Usage // Cheese Industry. 2000, 2, 28-29.

11. Kryuchkova, V.V. Prebiotics in Functional Fermented Milk Products // Dairy Products Industry. 2009, 7, 34-36.

12. Leonidov, D.S. Lactulose: Range of Use in Food Industry // Candy Manufacture and Breadbaking. 2011, 10, 34-35.

13. Leonidov, D.S. Prebiotics: Development Strategy for "Health Products" // Milk Processing. 2011, 9, 66-67.

14. Leonidov, D.S. Lactulose Prebiotic: Effective Strategy of Healthy Diet Development // Food 
Ingredients. Raw Materials and Additives. 2011, 2, 37-39.

15. Serov, A.B.; Ryabtseva, A.B. // Bulletin of North Caucasian State Technical University. 2006, 3, 78-82.

16. Afifi, H.S.; Shelaibi, A.A.A.; Laleye, L.C. Measurement of thermal characteristics of spray-dried milk and juice blend / // International journal of food sciences \& nutrition. 2009, 60 (7), 89-98.

17. Andrews, G.R. Lactulose content, color and the organoleptic assess-ment of ultra heat treated and sterilized milks // J.of Dairy Research. 1987, 54, 493-507. 